\title{
Vom Business Case zum Public Case! \\ Frosch- und Vogelperspektiven zur Global Governance
}

\section{THOMAS BESCHORNER}

Korreferat zum Beitrag von Klaus Dieter Wolf und Sandra Schwindenhammer

(Groß-)Unternehmen im 21. Jahrhundert sind nicht mehr nur „quasi-öffentliche Institutionen" (Ulrich 1977), sondern governance-politische Akteure, die sich aktiv in die Gestaltung der Spielregeln einbringen. Klaus Dieter Wolf und Sandra Schwindenhammer (2011) gehen in dem vorliegenden Beitrag der Frage nach, welches Potential private transnationale Selbstregulierungen als neue gesellschaftliche Steuerungsmechanismen unter den Bedingungen einer Weltgesellschaft aufweisen. Ihr Augenmerk richten sie dabei auf Legitimationsaspekte. Nicht die Frage der Effizienz, sondern normative Anforderungen an diese noch neuartigen Regime stehen damit im Mittelpunkt der wertvollen Untersuchung der Autoren. Diese Fragestellung ist deshalb von hervorragender Relevanz, weil „eine deutliche Lücke zwischen wahrscheinlichen Formen transnationaler privater Selbstregulierung auf der einen Seite und normativ wünschenswerten Formen auf der anderen klafft" (Wolf/Schwindenhammer 2011: 12). Klaus Dieter Wolf und Sandra Schwindenhammer argumentieren in ihrem Beitrag, dass ein „Entweder-Oder“ (Wolf/Schwindenhammer 2011: 24) von öffentlichen und privaten Regulierungen weder faktisch zutrifft noch hinsichtlich einer normativ gewünschten Praxis zielführend ist. Notwendig ist eine „institutionelle Konstitutionalisierung", in meinen Worten eine stärkere Hybridisierung von öffentlichen und privaten Regulierungen, die jenseits des Nationalstaates auch supranationale und internationale Politikebenen einschließen kann (Wolf/Schwindenhammer 2011: 24-25).

Ich möchte meinen Kommentar, bei genereller Zustimmung zu den Überlegungen von Wolf und Schwindenhammer, in aller gebotenen Kürze auf drei Aspekte beschränken: Es soll erstens vorgeschlagen werden, präziser zwischen einer einzelwirtschaftlichen und einer kollektiven Normenebene zu unterscheiden. Ich möchte zweitens anregen, den Erklärungsansatz für private Regulierungen um eine „Vogelperspektive“ zu ergänzen. Damit, so deutet sich drittens an, betritt - jenseits vom „business und public case“ - eine weitere wichtige Akteursgruppe die Arena: zivilgesellschaftliche Organisationen.

Ad (i): Wolf und Schwindenhammer wenden im Abschnitt 3.2 ihres Beitrages ihre vorangegangenen Überlegungen auf zwei Fälle an, indem sie zwei „Legitimationsbilanzen“, nämlich einmal auf einen Verhaltenskodex bei Shell und

Prof. Dr. Thomas Beschorner, Universität St. Gallen, Institut für Wirtschaftsethik, Tannenstrasse 19, CH-9000 St. Gallen, Tel.: +41 (0)71 - 224 3143, Fax: +41 (0)71 - 224 2881, E-Mail: thomas.beschorner@unisg.ch, Forschungsschwerpunkte: Wirtschafts- und Unternehmensethik, Handlungs- und Institutionentheorien. 
einmal auf die GRI beziehend, gegenüberstellen. Die Sinnhaftigkeit dieses „Vergleiches“ und die Frage, was wir darüber generell lernen können, erschließt sich mir leider nicht. Es mag zwar sein, dass die entwickelten Kriterien der Responsivität, der Selbstbestimmung, der Rechenschaftspflichtigkeit und der Autorität nicht nur auf (kollektive) Governance-Mechanismen, sondern auch auf Fragen der (individuellen) Unternehmensverantwortung angewendet und daraus wichtige Orientierungen hergeleitet werden können (z.B. eine starke Inklusion von Stakeholder-Interessen); für einen wirklichen Vergleich wären gleichwohl Betrachtungen auf derselben Analyseebene (entweder individuell oder kollektiv oder verschiedene (hybride) Steuerungsmechanismen) interessanter und aussagekräftiger.

Ad (ii): In dem Beitrag wird der Versuch unternommen, einen Erklärungsansatz für private Regulierungen zu entwickeln, in dem auf den „Business Case“ abgestellt wird. Die Autoren argumentieren hier differenziert, denn sie ziehen für die Erklärung, warum sich Unternehmen als „Normunternehmer“ engagieren, nicht nur im engeren Sinne ökonomische Argumente heran (Reputation, „license to operate“ usw.), sondern integrieren auch kulturwissenschaftliche Perspektiven („Sozialisation im Herkunftsland“, „unternehmensinterne, habitualisierte Kultur der Verantwortung“" etc.) (Wolf/Schwindenhammer 2011: 15). Daraus resultieren dann die „wahrscheinlichen Formen transnationaler privater Selbstregulierung" (Wolf/Schwindenhammer 2011: 11), denen später normative Perspektiven gegenübergestellt werden.

Die Einbeziehung einer einzelwirtschaftlichen (Frosch-)Perspektive für die Erklärung privater Regime ist zweifellos wichtig und notwendig, aus meiner Sicht aber nicht hinreichend. Zu ergänzen wäre die Betrachtungsweise um die Analyse gesamtgesellschaftlicher (System-)Zusammenhänge, bei denen nicht „Selbstrefentialitäten“ und die Eigenlogiken von Subsystemen, sondern die Konflikte und Spannungen zwischen unterschiedlichen Wertsphären (z. B. Wirtschaft, Politik, Zivilgesellschaft, Religion, Wissenschaft) herausgearbeitet werden, die wir faktisch beobachten können. Aus diesen Spannungen, so die Vermutung, resultieren neue institutionelle Arrangements, beispielsweise private Selbstregulierungen, die gewissermaßen vermittelnd zwischen den verschiedenen gesellschaftlichen Wertsphären wirken. Gekennzeichnet sind diese Institutionen dadurch, dass die exklusive Zuordnung zu nur einer Wertsphäre schwer fällt. Die „wahrscheinlichen Formen“ erklären sich aus meiner Sicht nicht nur aus einer einzelwirtschaftlichen Logik heraus, sondern primär aus spezifischen gesellschaftlichen Konstellationen bzw. Veränderungen dieser Konstellationen (makroskopische Betrachtung), die dann zu Konsequenzen auf der Mikroebene der Akteure führen, z.B. auch bei Unternehmen. Mit Bezug auf die Rolle von Unternehmen ist aus meiner Sicht derzeit nicht pauschal zu entscheiden, ob es sich bei ihren Reaktionen lediglich um Taktiken zur Abfederung von Verantwortungszuschreibungen durch die Gesellschaft handelt oder ob tatsächlich so etwas wie eine „Zwecktransformation“ stattfindet.

Ad (iii): Die Argumentation von Klaus Dieter Wolf und Sandra Schwindenhammer oszilliert zwischen „Business Case“ und „Public Case“. Ersterer bezieht sich auf das einzelwirtschaftliche Interesse von Unternehmen, letzter auf allgemeine gesellschaftliche Interessen (Gemeinwohl), wobei diese Gemeinwohlorientierung primär (neo-)westfälisch durch „Rechtsstaatlichkeit“ (wenn auch möglicherweise über den

zfwu 12/1 (2011), 29-31 
Nationalstaat hinausgehend) realisiert werden soll. Aus meiner Sicht wäre es interessant, diese wichtigen Überlegungen um weitere Perspektiven zu ergänzen, die in besonderem Maße die Rolle von zivilgesellschaftlichen Organisationen betreffen, deren prinzipielle Zweckbestimmung ebenso - wie bei staatlichen Organisationen die Gemeinwohlorientierung ist und die für sich „sowohl moralische als auch wissensbasierte Autorität [beanspruchen]" (Wolf/Schwindenhammer 2011: 4). Im Sinne dieses klassischen Dreiklangs von Staat, Wirtschaft und Zivilgesellschaft will ich mit vier Forschungsfragen anregen: Was erwarten wir von den zivilgesellschaftlichen Akteuren hinsichtlich privater Selbstregulierungen (jenseits von UnternehmensWatch-Dogs-Funktionen) konkret? Welche Kooperationsformen zwischen NGO und Unternehmen sind diesbezüglich aussichtsreich? Wie und inwieweit verändert sich die Rolle von Politik als Regelsetzer hin zu einer Moderatorin von deliberativen Prozessen? Letztlich und insgesamt: Wie kann eine zivilisiert Marktwirtschaft gelingen?

\section{Literaturverzeichnis}

Ulrich, P. (1977): Die Großunternehmung als quasi-öffentliche Institution. Eine politische Theorie der Unternehmung, Stuttgart: Poeschel.

Wolf, K. D./ Schwindenhammer, S. (2011): Vom Business Case zum Public Case? Der Beitrag privater Selbstregulierung zu Global Governance, in: Zeitschrift für Wirtschaft- und Unternehmensethik, Jg. 12/Heft 1, 10-28. 